\title{
ER stress drives obesity by reducing energy expenditure
}

Although endoplasmic reticulum (ER) stress and metabolic inflammation are associated with the development of obesity, whether the two processes converge to disrupt energy homeostasis during progression of the disease was uncertain. Now, Shan et al. show that the inositol-requiring enzyme 1 $\alpha$ (IRE1 $\alpha$; an ER stress sensor) pathway in adipose tissue macrophages (ATMs) drives the progression of obesity and the metabolic syndrome by reducing energy expenditure.

Having first shown that a high-fat diet (HFD) induced ER stress (demonstrated by activation of IRE1 $\alpha$ ) in parallel with adipose-tissue inflammation in mice, the researchers generated myeloid (bone-marrow-derived macrophage and peritoneal macrophage)-specific IRE1 $\alpha$-deficient mice to investigate possible metabolic actions of the IRE1 $\alpha$ pathway during overnutrition. Remarkably, myeloid IRE1a deficiency prevented obesity, insulin resistance, hyperlipidaemia and hepatic steatosis induced by a HFD. These metabolically beneficial effects were accompanied by increases in brown adipose tissue activity, white adipose tissue browning and energy expenditure. Moreover, IRE1 $a$ deficiency increased M2 (anti-inflammatory) polarization of ATMs.

The findings suggest that, during overnutrition, IRE1 a increases adipose tissue inflammation by guiding ATM polarization and drives obesity and the metabolic syndrome by impairing the thermogenic capacity of adipose tissue and thus energy expenditure. "Targeted modulation of IRE1 $\alpha$ activity in ATMs might open new avenues for developing brown- and beige-fatenhancing therapeutics to treat obesity and metabolic disease," conclude the authors.

David Holmes

ORIGINAL ARTICLE Shan, B. et al. The metabolic

ER stress sensor IRE1 1 suppresses alternative activation of macrophages and impairs energy expenditure in obesity. Nat. Immunol. http://dx. doi.org/10.1038/ni.3709 (2017)

FURTHER READING Baiceanu, A. et al.

Endoplasmic reticulum proteostasis in hepatic steatosis. Nat. Rev. Endocrinol. 12, 710-722 (2016) 